\title{
"Scrivere la storia significa incasinare la geografia": mappe postcoloniali
}

\section{"Writing History Means Playing Havoc With Geography": Postcolonial Maps}

\author{
Chiara Mengozzi [chiara.mengozzi@gmail.com] \\ Univerzita Karlova v Praze, Repubblica Ceca \\ CEFRES - Praha, Repubblica Ceca \\ Univerzita v Hradci Králové, Repubblica Ceca
}

\section{Riassunto}

Dopo aver precisato il ruolo delle mappe nel quadro dei progetti espansionistici dell'Europa e i diversi usi di questo topos nella letteratura (post)coloniale, il contributo si focalizza su due opere emblematiche, che condividono la stessa ipotesi di fondo, ovverosia l'idea che la storia individuale e collettiva siano strettamente correlate alla cartografia del territorio urbano e nazionale: La mia casa è dove sono di Igiaba Scego e Maps di Nuruddin Farah. Si dimostrerà che, mentre il romanzo di Scego giunge a una proposta identitaria conciliatoria basata sulla fiducia nella narrazione come strumento "per rendere conto di sé" e su una disinvolta equivalenza tra l'"io" e il "noi"; quello di Farah, concependo il rapporto tra l'individuale/familiare e il collettivo/nazionale secondo le modalità dell'allegoria e facendo del protagonista un narratore inaffidabile, approda a una convincente critica della narrazione come privilegio delle elite metropolitane, e delinea una nuova geografia di rapporti interpersonali e internazionali dove le frontiere risultano completamente denaturalizzate.

\section{Parole Chiave}

Letteratura Postcoloniale; Letteratura della migrazione; Nuruddin Farah; Igiaba Scego; Identità; Narrazione; Mappe; Geografia; Focalizzazione; Città

\begin{abstract}
After having defined the role of the maps within European expansionist plans and the different uses of this topos in (post)colonial literature, my paper will focus on two emblematic novels, which share the same basic assumption, namely the idea that history, both individual and collective, is closely related to the mapping of urban and national territory: La mia casa è dove sono written by Igiaba Scego, and Maps by Nuruddin Farah. I will demonstrate that 1) Scego's novel leads to a conciliatory construction of identity based on the confidence in narratives as a mean to "give an account of oneself" and on un unwarranted equivalence between "I" and "We" 2) Nuruddin Farah's Maps, on the contrary, conceives the relation between individual and collective or between being member of a family and being member of a nation in allegorical way and adopts the point of view of an unreliable narrator. This way, he provides a convincing criticism of narrative as a privilege reserved to the members of the wealthy metropolitan elite, and
\end{abstract}


sketches out a new geography of interpersonal and international relations where the frontiers are wholly de-naturalized.

\title{
KeYWORDS
}

Postcolonial Literature; Migrant Literature; Nuruddin Farah; Igiaba Scego; Identity; Narration; Maps; Geography; Focalization; City

RICEVUTO 2015-08-31; ACCETTATO 2015-12-22

\begin{abstract}
Just as none of us is outside or beyond geography, none of us is completely free from the struggle over geography. That struggle is complex and interesting because it is not only about soldiers and cannons but also about ideas, about forms, about images and imaginings. (Edward Said, Culture and Imperialism)
\end{abstract}

Visto il ruolo preminente assunto dalla cartografia nel quadro dei progetti espansionistici dell'Europa, non sorprende che le mappe - siano esse fisicamente incorporate nel testo, descritte come in una sorta di ekphrasis, o implicite, cioè sottese al racconto e desumibili dal lettore "chiamato a costruirsi una mappa mentale dei luoghi nominati" (Guglielmi - Iacoli 2012: 15) ${ }^{1}$ - costituiscano un motivo ricorrente, se non un vero e proprio tema ${ }^{2}$ o $\operatorname{arcitema}^{3}$ trasversale a moltissima letteratura coloniale tra Ottocento e Novecento, e presente tanto nei grandi classici dell'epoca quanto nelle opere propagandistiche e di largo consumo.

Come nel celebre romanzo di Conrad, Heart of Darkness, la mappa del mondo con i suoi vuoti da riempire offre al giovane Marlow “promesse di conoscenza e potere" (Iacoli 2008: 31), anche nei molto più modesti romanzi coloniali italiani fascisti degli anni Venti e Trenta - oggi pressoché sconosciuti, ma al tempo noti anche al grande pubblico ${ }^{4}$ - il motivo della mappa

1 Faccio qui riferimento all'utile proposta tassonomica di Iacoli (2007). Huggan (1994), invece, distingue tra mappe usate come icona, motivo e metafora. I termini della ripartizione, tuttavia, non sono equiparabili, ed è per questo che la proposta non convince interamente. Mentre i primi due riguardano le modalità di inserzione della mappa nel testo letterario, il terzo concerne il senso attribuitole dal lettore. Tanto le illustrazioni, quanto le mappe descritte o nominate nel testo possono evidentemente essere convocate in senso simbolico o metaforico.

2 La distinzione tra tema e motivo, non sempre agevole, è affrontata in maniera chiara e convincente da Giglioli (2001). Mentre il motivo “è un'unità di segmentazione del contenuto" (20) e appartiene dunque al dominio dell'argomento, il tema non appartiene né all'argomento né al senso, ma è piuttosto la negoziazione continua tra i due che ha luogo nell'atto della lettura. Se il motivo è statico (è cioè un'unità isolabile del testo) e oggettivo, il tema è dinamico (cioè costruito dal lettore) e mutevole. Benché né Iacoli né Huggan distinguano esplicitamente tra mappe/motivi e mappe/temi, le mappe implicite sono evidentemente il risultato di un atto ermeneutico (la tematizzazione) e spetta interamente al lettore tracciarle.

3 Sull'uso di “arcitema”, cfr. Bertoni - Fusillo (2001). Gli arcitemi, come il viaggio o l'eros, per citare soltanto due tra i più antichi e diffusi nella storia della letteratura occidentale, si prestano a infinite realizzazioni e aggregano a loro volta temi ulteriori. Nei romanzi che analizzerò a breve, la mappatura del territorio urbano e nazionale aggrega diverse questioni, dal problema dell'identità e della narrazione di sé al tema della frontiera.

4 Tra gli autori coloniali attivi tra gli anni Venti e Trenta più noti al grande pubblico si annoverano Arnaldo Cipolla, Mario de' Gaslini, Guido Milanesi, Mario Appelius, Gino Mitrano Sani, Vittorio Tedesco Zammarano. Sul romanzo coloniale italiano, cfr. almeno Pagliara (1991 e 2001), Tomasello (2004: 120-76), Bonavita (2009: 29-106), Boddi (2012). 
come metafora di conquista e possesso è ampiamente sfruttato. Pochi specialisti forse ricorderanno l'incipit di Azanagò non pianse, romanzo di Vittorio Tedesco Zammarano, pubblicato nel 1934: "L'ufficiale curvo sul tavolo da lavoro, disegnava sul rilievo topografico della zona di confine piccoli cerchi rosso - bianco - verdi lungo il tracciato della sua ultima escursione; e con la fantasia vedeva già tali segni convenzionali tramutarsi in emblemi di effettiva occupazione e di saldo dominio italiano" (1934: 2). Un'opera di propaganda razzista e coloniale, stilisticamente mediocre e ideologicamente deprecabile, ma nondimeno interessante in questa sede perché in poche righe esplicita la posta in gioco principale della cartografia dell'epoca, evocando il duplice ruolo assunto dalle carte coloniali, al contempo strumenti di descrizione funzionali alla conquista (la dominazione politica e il controllo militare di un territorio sono necessariamente preceduti dall'appropriazione simbolica dello stesso) e potenti mezzi di persuasione (le carte coloniali non si limitano a rendere possibile o a notificare la conquista, ma hanno anche il compito di promuovere, legittimare e celebrare l'idea di impero $)^{5}$.

Gli scrittori postcoloniali ereditano la vocazione cartografica che aveva caratterizzato la produzione di epoca coloniale, invertendola però di segno. Essi risemantizzano il topos della mappa nel tentativo di decolonizzarla (Huggan 1989). Questa sorta di "write back cartografico"6 prevede tre principali operazioni: 1) l'uso parodico, ironico della mappa 2) la proposta di mappe o topografie urbane e nazionali alternative e 3), infine, una riflessione più o meno esplicita sullo statuto metaforico della mappa. Quest'ultima, nelle tre varianti sopraindicate (la mappa incorporata, descritta o implicita) è convocata non più (o non soltanto) come metafora di controllo e possesso, come accadeva spesso nella letteratura coloniale, ma piuttosto come metafora della "rappresentazione" e della sua convenzionalità, come paradigma del rapporto arbitrario tra segno e referente, tra significante e significato, poiché la mappa, si sa, non è il territorio, benché si presenti come una sua copia fedele e oggettiva; al contrario, proprio come uno specchio, la mappa riflette l'ideologia, i desideri, le proiezioni di chi la disegna.

Nelle pagine che seguono propongo la lettura comparativa di due romanzi che presentano una relazione intertestuale forte, finora non analizzata dalla critica con sufficiente attenzione ${ }^{7}$ : La mia casa è dove sono (2010), opera di ispirazione autobiografica firmata da Igiaba Scego, nata a Roma da genitori somali, e Maps, pubblicato nel 1986 da Nuruddin Farah, probabilmente il più importante scrittore della diaspora somala ${ }^{8}$.

$5 \quad$ Sul potere delle mappe, cfr. Harley (1988), Wood, Fels (1993), Black (1997) e in particolare i primi due capitoli Cartography as Power e Mapping the World and its People. Sulla cartografia coloniale italiana, cfr. Atkinson (1995) e Casti (2003 e 2007).

6 Lespressione da me scelta è chiaramente modellata sul famoso titolo del libro di Ashcroft, Griffith e Tiffin, The Empire Writes Back.

7 Nei testi critici relativi alle scrittrici postcoloniali italiane (Igiaba Scego, Cristina Ali Farah, Gabriella Ghermandi, Shirin Ramzanali Fazel, per citare le principali) compare spesso il nome di Nuruddin Farah come termine di riferimento. Tuttavia, nella maggior parte dei casi, ci si limita a dei richiami a Yesterday, Tomorrow (2000), libro in cui Farah raccoglie le testimonianze della diaspora somala nel mondo. Per una comparazione tra le scrittici di origine somala attive in Italia e la produzione romanzesca di Farah, vedi Proglio (2011: 120-64).

8 Scego cita in esergo del testo citato un brano tratto da Yesterday, Tomorrow (nella traduzione italiana Rifiugiati. Voci della diaspora somala) di Nuruddin Farah: "Ho preso dimora in un territorio dai confini incerti che sono solito definire il paese della mia immaginazione", passaggio che a sua volta rimanda alle "patrie immaginarie" di cui parla Rushdie (1991). Al contrario, il debito nei confronti di Maps, romanzo dal quale l'autrice trae a mio avviso il motivo di fondo del suo libro (il disegno/mappa di Roma e Mogadiscio), non è esplicitamente dichiarato. 
I due romanzi sono per certi versi analoghi, per altri speculari. Analogo è il trattamento della mappa, sia descritta sia implicita, così pure l'ipotesi sottesa al racconto, vale a dire l'idea che la storia individuale e collettiva siano strettamente correlate alla cartografia del territorio urbano e nazionale. Speculare è certamente la cornice degli avvenimenti: mentre il primo è ambientato a Roma, ma è abitato "spettralmente" da Mogadiscio e dalle colonie italiane, il secondo si svolge in Ogaden e a Mogadiscio, ma questi luoghi riflettono anche i sogni di conquista coloniale e neocoloniale delle potenze straniere. Altrettanto capovolto è il rapporto che lega i rispettivi protagonisti alle mappe: mentre Igiaba (qui convocata come personaggio del testo), con leggerezza e ironia, usa la mappa come espediente per una presa di coscienza identitaria (individuale e collettiva) e cerca di immaginare una nuova topografia dell'Urbe capace di includere le vite e i percorsi di migranti, rifugiati e marginali, le carte disegnate dal giovane Askar (che coltiva il sogno della Grande Somalia durante la guerra con l'Etiopia per il controllo dell'Ogaden) sembrano anacronisticamente rilanciare il ruolo della cartografia coloniale come strumento di descrizione, conquista e persuasione.

L'atteggiamento e le intenzioni dei protagonisti, però, sono a mio avviso speculari ai risultati cui approdano i romanzi nel loro complesso. Detto altrimenti, un'attenta analisi comparativa e qui anticipo alcune delle conclusioni del mio contributo - permetterà di dimostrare che, mentre il romanzo di Scego giunge a una proposta identitaria tutto sommato conciliatoria (presente peraltro in molta letteratura migrante o postcoloniale $)^{9}$, basata su una fiducia nella narrazione come strumento "per rendere conto di sé" 10 , su una disinvolta e indebita equivalenza tra l'“io" e il "noi", sulla traduzione e l'esaltazione del meticciato come strategia di (auto)legittimazione dello scrittore migrante; quello di Nuruddin Farah, al contrario, concependo il rapporto tra l'individuale/familiare e il collettivo/nazionale secondo le modalità dell'allegoria, facendo del protagonista un narratore inaffidabile e alternando il racconto in prima, seconda e terza persona, approda a una convincente critica della narrazione come privilegio delle elite migranti metropolitane, e delinea una nuova geografia di rapporti interpersonali e internazionali dove le frontiere risultano completamente denaturalizzate.

Nel primo capitolo de La mia casa è dove sono, Scego racconta la scena narrativa che rappresenta loccasione da cui nasce il romanzo, ovvero una riunione (reale o fittizia, poco importa) tra i membri della sua famiglia in diaspora avvenuta a Manchester a casa del fratello, durante la quale i protagonisti si abbandonano ai "ricordi della [loro] vecchia terra" (2010: 12), la Somalia, che li porterà a disegnare una mappa di Mogadiscio ${ }^{11}$, ormai distrutta da anni di guerre civili,

9 Basti pensare alla frequenza con cui scrittori e scrittrici migranti attribuiscono un valore terapeutico alla scrittura di sé e alla ricorrenza del lieto fine che spesso coincide con la decisione di iniziare a narrare la storia che abbiamo appena finito di leggere. Su questi e altri topoi presenti nelle scritture migranti, cfr. Mengozzi (2013b).

10 Diversi filosofi, tra cui Cavarero (1997) e Ricœur (1985), sottolineano l'importante ruolo della narrazione nella costruzione dell'identità personale: il racconto permetterebbe infatti di articolare la dialettica tra il "cosa" e il "chi" dell'identità. Judith Butler, tuttavia, nel suo Giving an Account of Oneself (2005), dimostra in maniera convincente che "raccontare una storia su di sé non equivale a rendere conto di sé" (22): a) nella vita di un soggetto ci sono aspetti che si sottraggono alla consequenzialità di un racconto b) il soggetto si trova costitutivamente nell'impossibilità di rendere conto appieno di ciò che lo determina in quanto tale $\mathrm{c}$ ) l'accesso alla narrazione è codificato da norme che "decidono in anticipo chi diventerà o meno un soggetto" (18) legittimato a prendere la parola e a raccontare una storia su di sé. Per una critica dell'identità narrativa e della celebrazione della narrazione in molta letteratura (e critica) contemporanea, cfr. anche Meneghelli (2013).

11 Il disegno di Mogadiscio ritorna nel documentario La quarta via: Mogadiscio, Pavia, tratto da un racconto orale di Kaha Mohamed Aden e diretto da Brioni, Guida e Chiscuzzu. La protagonista, dopo aver annunciato che per lei è 
ma ancora integra nella loro memoria. Igiaba ben presto si accorge che quella mappa non è completa perché la sua città, come le fa notare giustamente sua madre, non è (o non è soltanto) Mogadiscio, ma anche e soprattutto Roma, dove è nata e dove ha trascorso gran parte della sua vita. Che cosa fa, dunque? Ci racconta di aver acquistato dei post-it ("non volevo un foglio di carta - afferma la narratrice - volevo qualcosa di provvisorio e scomponibile" (34)) e di averli appiccicati sul disegno di Mogadiscio scrivendo su ognuno di essi i nomi di quartieri, piazze e monumenti di Roma. Il risultato è un gran caos, ma la mappa è "finalmente completa" (ibid.).

A quel punto il romanzo si avvia una seconda volta. La mappa raccontata cede il passo a una mappa implicita; dal disegno di Mogadiscio sul quale si ammonticchiano disordinatamente ricordi e vicende personali che legano Igiaba Scego a Roma, si passa alla città di Roma percorsa dalla protagonista in cerca delle tracce di quello che considera il passato rimosso dell'Italia, ovverosia il colonialismo. Come in una sorta di parodia delle guide turistiche di Roma, ogni capitolo è dedicato a un noto luogo o monumento della capitale (il teatro Sistina, Piazza Santa Maria Sopra Minerva, Piazza di Porta Capena, Roma Termini ecc.), ma ognuno di essi viene investito di un senso nuovo, diventa il teatro di racconti individuali, familiari e collettivi legati alla memoria del colonialismo italiano e dei suoi effetti a lungo termine (l'immigrazione somala in Italia per esempio) ${ }^{12}$. Attraverso questi racconti, la narratrice traccia una nuova carta dell'Urbe, fatta di traiettorie transnazionali nell'intento di rendere, agli occhi dei lettori italiani, Mogadiscio un po' più familiare e Roma un po' più spaesante.

La proposta di Scego è indubbiamente suggestiva e La mia casa è dove sono è senz'altro il più riuscito e godibile dei suoi romanzi ${ }^{13}$. Restano però delle questioni aperte che provo a formulare sinteticamente tramite l'analisi della conclusione di cui riporto un estratto:

Che significa essere italiano per me... Una domanda che batteva come un viandante sconosciuto alla porta di casa: io ho provato a scriverla una risposta. Essere italiano per me...

[...] Non avevo una risposta. Ne avevo cento. Sono italiana, ma anche no. Sono somala, ma anche no.

Un crocevia, uno svincolo.

Un casino, un mal di testa.

Ero un animale in trappola.

Un essere condannato all'angoscia perenne.

Poi mi sono ricordata di un racconto di Karen Blixen.

$[\ldots]$

molto difficile raccontare Mogadiscio, prende carta e penna e traccia con un ordinario pennarello, su un semplice foglio di carta, quattro vie di colori diversi, come a cercare un appiglio materiale per la memoria frammentata di una città semidistrutta. Il racconto prende avvio proprio da questo gesto.

12 L'autrice, insieme a Rino Bianchi, pubblicherà nel 2014 un volume dal titolo Roma negata dove, attraverso un'alternanza tra parole e immagini, si intendono raccontare i luoghi (rimossi) della capitale connessi, direttamente o indirettamente, al colonialismo italiano. Anche il motivo della passeggiata per le strade di Roma, presente ne La mia casa è dove sono, ritorna in Roma negata: "Cammino.... Lo faccio sempre quando ho qualche pensiero. Cammino per le strade trafficate della mia Roma Capoccia [...]” (13).

13 Oltre Babilonia (2008) è probabilmente un'opera di maggior impegno, se non altro per la mole e l'alternanza di numerose voci narranti. Tuttavia, vi rientra fin troppo materiale, dal discorso queer a quello postcoloniale, dall'identità black a quella religiosa e musulmana, dalla diaspora somala alla dittatura argentina, ecc. Sul romanzo di Scego come "feticizzazione del postcoloniale", come programmatica, quasi manualistica "mise en abyme dell'intero discorso postcoloniale”, cfr. Pinzuti (2012: 202). Sugli aspetti linguistici e retorici della produzione di Scego, cfr. Sirotti (2013). 
Mi aveva colpito il titolo Il racconto del Cardinale. Ricordo che una signora chiedeva al Cardinale: "Ma tu chi sei?", e a questa domanda "Chi sei?" il Cardinale ribatteva: «Risponderò con una regola classica: racconterò una storia».

Era questa la chiave.

Era inutile cercare di riempire i punti di sospensione delle definizioni.

Era una battaglia persa in partenza.

Quei puntini ci avrebbero perseguitato per tutta la vita.

Era meglio fare come il Cardinale: provare a raccontare il percorso che si era fatto fino a quel momento e forse i percorsi di chi sentiamo veramente vicini.

Io ho provato qui a raccontare brandelli della mia storia. [...]

Mi sono concentrata sui primi venti anni della mia vita perché sono stati i venti anni che hanno preparato il caos somalo [...] Ma sono stati anche i venti anni in cui l'Italia è cambiata come non mai. [...]

Io sono il frutto di questi caos intrecciati.

E la mia mappa è lo specchio di questi anni di cambiamenti.

Non è una mappa coerente. È centro, ma anche periferia. È Roma, ma anche Mogadiscio.

È Igiaba, ma siete anche voi. (pp. 158-60)

Pur nel tentativo apprezzabile di creare una storia condivisa tra l'Italia e le sue colonie (Igiaba Scego, nell'ultima riga del libro, consegna il racconto ai lettori, invitandoli a pensare che la sua personale storia di vita sospesa tra Roma e Mogadiscio li riguardi da vicino), il brano (ma il romanzo in generale) presenta, a mio avviso, tre punti critici che credo essere comuni a molta letteratura migrante o postcoloniale ${ }^{14}$.

1) Il primo problema è il "pathos della lacerazione" che si risolve nel "pathos dell'entre-deux"15. Scego segue un canovaccio narrativo ormai consunto e presente in moltissimi testi scritti dalle cosiddette seconde generazioni. Questa produzione propone in maniera ricorrente un conflitto identitario tra due metà che sembrano escludersi a vicenda (nel caso di Scego, la sua metà somala e quella italiana), come se l'identità di una persona fosse divisibile in due sfere dai contorni precisi, come se le persone passassero davvero il tempo a incasellare i propri gesti, abitudini, usanze, credenze in taluna o talaltra "cultura"16. Dopo aver inscenato il conflitto, giunge immancabile la soluzione: nel libro di Scego - così come in gran parte delle narrazioni autobiografiche

14 Ho analizzato anche in altra sede il racconto di Igiaba Scego, mettendo l'accento però su altri aspetti, in particolare ciò che definisco la "scena interlocutoria della narrazione personale", topos narrativo onnipresente nel corpus della letteratura (italiana) della migrazione. Nel mio libro dedicato a questa produzione, definivo il romanzo di Scego quasi una sorta di manifesto della letteratura cosiddetta migrante (cfr. Mengozzi, 2013a: 132-6). Su questo punto, la mia opinione non è cambiata, ma il confronto con Farah mi ha permesso di far emergere anche i limiti del testo di Scego e di formulare una critica della "mondialità" offerta dalle élite migranti metropolitane.

15 Traggo queste due felici espressioni da un articolo sulla letteratura postcoloniale francofona di Dumouillé (2012).

16 Nel racconto di ispirazione autografica Salsicce (Scego, 2005), l'apparente conflitto identitario viene inscenato attraverso due elenchi separati dei momenti in cui la narratrice si sente somala e di quelli in cui si sente italiana. 
o autofinzionali delle seconde generazioni ${ }^{17}$ - i personaggi scoprono progressivamente che non è il caso di lacerarsi; al contrario, in questo mondo sempre più meticcio e creolizzato, invece di insistere sulla duplice esclusione, essi possono tranquillamente rivendicare con orgoglio la propria duplice o molteplice appartenenza.

Come dar loro torto. Il punto è che l'esaltazione dell'ibrido è diventata un'ideologia perfettamente digerita e, anzi, rifunzionalizzata dalle logiche del mercato globale che si nutre di differenze per moltiplicare i target (Hardt - Negri 2000). La narratrice del romanzo di Scego pare quasi compiacersene, per esempio quando racconta le sue esperienze scolastiche e ci dice di essere stata discriminata dai compagni di classe per il colore della sua pelle, per poi rendersi conto che, grazie all'appoggio di un'illuminata maestra elementare, progressivamente iniziava a occupare il centro dell'attenzione richiamando gli sguardi e l'interesse dei compagni: "Avevo più successo del mago Zurlì. E a poco a poco cominciai ad avere amici e a essere glamour" (Scego 2010: 156). Certo, meglio essere alla moda che oggetto di insulti, ma, ahimè, è difficile leggere questo ribaltamento (dall'esclusione delle differenze alla loro estetizzazione) soltanto in senso liberatorio. Il percorso raccontato da Scego corrisponde, in fondo, all'ambivalenza delle letterature migranti e postcoloniali nel sistema letterario mondiale contemporaneo: un contro-discorso che rischia di essere interamente assorbito dalle logiche del mercato globale.

2) Il secondo punto critico del brano citato riguarda la disinvolta equivalenza tra l"io" e il "noi". Se nella conclusione, che a prima vista ha tutta l'aria di essere un discorso soltanto personale, può fare irruzione improvvisamente e inaspettatamente il plurale ("Quei puntini ci avrebbero perseguitato tutta la vita") è perché in tutto il testo Scego alterna senza soluzione di continuità il piano individuale della narrazione personale a quello familiare e collettivo. In altre parole, la sua Roma e la sua Mogadiscio finiscono per diventare la Roma e la Mogadiscio di diverse collettività di emarginati di cui l'autrice sembra farsi portavoce. Questo "noi", così spesso convocato nel testo, cambia continuamente e aproblematicamente referente: "Noi" famiglia Scego, cioè membri dell'intellighenzia somala in diaspora ${ }^{18}$; "noi" vittime dirette o indirette del colonialismo ("noi che non abbiamo mai chiesto nulla a quest'Italia che ci ha colonizzato", 97), "noi” emigranti somali ("tutti noi della diaspora somala”, ibid.); "noi” somali di Roma ("una comunità invisibile come la nostra", ibid.) e, infine, "noi somali” tout court. Tutti questi "noi", nei quali Scego di volta in volta si colloca, pur così diversi, finiscono nel suo discorso per equivalersi, per essere tutti riuniti in un unico "paradigma vittimario" (cf. Giglioli, 2014) funzionale a legittimare il soggetto parlante o, meglio, scrivente, che sorvola comodamente sulle differenze (di classe per esempio), finendo per presupporre e ribadire di continuo quellopposizione che si vorrebbe superare tra la Somalia e l'Italia, tra "noi" e "voi", tra Roma e Mogadiscio, presentate invece in apertura come "gemelle siamesi separate alla nascita" (Scego 2010: 11).

17 Considerazioni simili si trovano in Quaquarelli (2010), a cui rimando per gli altri esempi tratti dal corpus migrante.

18 Il padre di Igiaba Scego, come racconta la stessa autrice nel libro, apparteneva alla classe di dirigenti somali scolarizzati in Italia e incaricati di guidare la Somalia nella fase del delicato passaggio dall'Ammistrazione fiduciaria italiana (A.F.I.S) all'indipendenza avvenuta nel 1960. 
3) Il terzo punto critico (strettamente legato al precedente) ha a che fare con la fiducia nella narrazione come strumento per "rendere conto di sé"19. Se è vero che alla domanda "chi sei" (o "chi siete?", nella sua variante collettiva) non si può rispondere con una definizione ("Inutile - ci dice Scego - cercare di riempire i punti di sospensione delle definizioni”), non è detto che l'identità narrativa, lo storytelling sia una pacifica soluzione, soprattutto se l'identità in questione oscilla tra l'individuale e il collettivo. Scego sembra non tener conto dello stretto legame tra narrazione e normatività e tra narrazione e autori(ali)tà. La narrazione di sé, infatti, come è ben dimostrato dal primo dei punti critici qui individuati (decine e decine di narrazioni migranti o postcoloniali che riproducono lo stesso schema, dal conflitto identitario alla pacifica "soluzione" ibrida o meticcia $)^{20}$, si basa ampiamente sull'uso ricorrente di topoi che la strutturano. Essa, inoltre, è regolata da norme che decidono preventivamente chi è un soggetto degno di prendere la parola: chi parla? Con quale autorità? A nome di chi lo fa? Domande cruciali che Scego non sembra porsi. Cruciali perché, senza ombra di dubbio, gli scrittori migranti e postcoloniali hanno trovato spazio legittimo nel contesto editoriale del primo mondo proprio mascherandosi - per scelta o imposizione - da informanti nativi, per interpretare (e tradurre) a uso, consumo e informazione del lettore occidentale i margini da cui provengono, gli echi del mondo, le tradizioni linguistiche e culturali dei paesi d’origine.

Le principali questioni sollevate grazie all'analisi del testo di Scego (1. la questione identitaria: chi sono? Chi siamo noi? Che cosa vuol dire essere somalo? Come si costruisce il rapporto tra spazi e identità? 2. Il problema della rappresentanza: chi parla e a nome di chi? 3. E infine i limiti della narrazione personale) sono anche al cuore del romanzo di Farah, ma, come vedremo, la "soluzione" - se di soluzione si può parlare - è molto diversa.

La storia raccontata nel romanzo si svolge nel 1977 durante la guerra combattuta tra l'Etiopia e la Somalia, intenzionata quest'ultima a recuperare un vasto territorio di frontiera noto con il nome di Ogaden, abitato in prevalenza da somali musulmani, ma assegnato dall'Organizzazione delle Nazioni Unite all'Etiopia nel $1954^{21}$. È utile ricordare che, alla fine dell'Ottocento, e in particolare dopo il 1869, anno dell'inaugurazione del canale di Suez, il Corno d'Africa cominciò ad attirare, per la crescente importanza strategica del Mar Rosso, gli interessi di tre stati europei (le due superpotenze coloniali, Francia e Inghilterra, e il neonato stato italiano in cerca di consolidamento), che si spartirono nel giro di pochi anni il territorio dei somali. Dopo

19 Cfr. la nota n. 10 del presente articolo.

20 È così in quasi tutti i testi della migrazione in italiano e nei romanzi delle "seconde generazioni" (vedi Ali Farah, Ghermandi, Scego, Abdel Quader, autrice del fortunatissimo Porto il velo e adoro i Queen, 2008). Fa eccezione Kuruvilla (2008 e 2012): nei suoi racconti e romanzi il conflitto identitario sembra non risolversi mai.

21 L'Ogaden diventa un oggetto di contesa già alla fine dell'Ottocento. Occupato dall'impero etiope sotto il regno di Menelik II (1897), questa regione di frontiera viene annessa alla Somalia italiana e inclusa nei territori dell'AOI (Africa Orientale Italiana) dopo la guerra contro l'Etiopia nel 1936. Nel 1941, i territori coloniali italiani passano sotto il controllo militare britannico. Dopo tredici anni di occupazione britannica, nel 1954, l'Ogaden torna all'Etiopia. Questa decisione è all'origine di un forte risentimento somalo e contribuirà a fomentare il programma politico pansomalo, nato già nell'immediato secondo dopoguerra nell'intento di riunificare in un unico stato-nazione tutti i somali, divisi tra ex Somalia britannica, ex Somalia italiana, ex Somalia francese, Ogaden e la parte nord-orientale del Kenya. Sulle complesse vicende del Corno d'Africa dalla decolonizzazione ai giorni nostri, cfr. il IV volume del monumentale Gli Italiani in Africa Orientale scritto dal più importante storico del colonialismo italiano, Del Boca (1984), e Guglielmo (2013), più agile e sintetico. 
il secondo conflitto mondiale, agli interessi delle antiche potenze coloniali si sommarono quelli sovietici e americani che intervennero a scuotere ulteriormente il già complicato quadro e che avrebbero portato ad assecondare, in un primo momento, soprattutto le rivendicazioni etiopi già espresse a Yalta nel 194522. L'Organizzazione delle Nazioni Unite nel 1950 stabilì non soltanto che l'Eritrea sarebbe stata federata all'Etiopia, ma anche che la Somalia sarebbe stata affidata per dieci anni a un'amministrazione fiduciaria italiana (A.F.I.S.) avente l'obiettivo di guidare il paese verso l'indipendenza. Il $1^{\circ}$ luglio 1960, la Somalia ottenne effettivamente l'indipendenza, ma senza tuttavia acquisire i territori rivendicati dal movimento pansomalo. Nell'attuale Somalia, infatti, confluirono soltanto l'ex Somaliland inglese e l'ex Somalia italiana, mentre l'Ogaden rimase all'Etiopia e la Somalia francese scelse l'indipendenza prendendo in seguito il nome di Repubblica di Gibuti.

Il romanzo di Farah - intessuto di metafore spaziali ${ }^{23}$ - ruota attorno alla possibilità/impossibilità di stabilire delle frontiere (tra le persone, le etnie, gli stati, le culture, le lingue) e presenta la storia individuale del protagonista e le relazioni tra i membri della sua famiglia come un'allegoria della storia nazionale somala ${ }^{24}$ nei suoi rapporti con altri popoli e culture, percepiti (dal protagonista in particolare) come unità discrete dai contorni individuabili, ma in realtà separati gli uni dagli altri da linee di demarcazione che si rivelano arbitrarie.

Il protagonista del romanzo, Askar, è un orfano di origini somale nato in Ogaden (quindi in territorio etiope) e cresciuto da Misra, una donna etiope di origini etniche miste, oromo e amare. Misra è la moglie-serva dello zio paterno di Askar (il brutale patriarca Qorax) e l'amante di Aw Adam, un uomo etiope di origine oromo, che riveste un ruolo importante nella comunità somala poiché è maestro coranico. Benché perfettamente somalofona e intimamente legata a una famiglia somala, Misra finirà per essere ingiustamente considerata come una straniera non soltanto dai membri della comunità somala, ma anche dal suo amatissimo figlio adottivo Askar. Se negli anni della prima infanzia, Askar si percepisce come "un'estensione del corpo di Misra" (Farah 2003: 100) e come "lo spazio che circondava la geografia del suo corpo" (ibid.), in seguito, al momento dello scoppio della guerra, egli si separa dalla donna, prima simbolicamente, sostituendo a una madre adottiva una madre collettiva (la Somalia) e poi concretamente, attraversando il confine tra l'Ogaden e la Somalia per giungere a Mogadiscio dai suoi zii materni, Hilaal et Salaado. Questi ultimi sono dei rappresentanti dellélite mogadisciana, ovverosia dell'intellighenzia somala educata nella lingua dell'ex-colonizzatore, l'italiano: "Askar annotava gli sforzi [dell'esercito somalo] sulla mappa che gli aveva regalato lo zio, proprio come tracciava,

22 Le principali priorità strategiche etiopi espresse dall'abile negus Hailé Selassié alla conferenza di Yalta furono per gran parte accolte: lo sbocco sul mare (tramite la federazione-annessione dell'Eritrea), il controllo sull'Ogaden, la partecipazione attiva ai vertici internazionali postbellici e gli accordi economici con gli Stati Uniti.

23 Ecco una lista parziale di esempi: "Lei (Misra) era il tuo spazio: ti muovevi sul suo corpo come un insetto si arrampica su un muro" (14); "Solo Misra poteva entrare nel mio spazio privato [...] aveva accesso al regno privato di cui ero signore assoluto" (37); "per anni, io contemplai il mondo dal trono sicuro della schiena di Misra (100); " Avevo un lenzuolo con cui coprirmi, che dovevo tenere a una certa distanza dalla ferita: di nuovo una questione di spazio, una questione di dettami geografici imposti dal dolore" (120); "Per prima cosa si coprì con un lenzuolo le cosce nude, su cui aveva tracciato la mappa del territorio fino allora riconquistato dai somali” (132)

24 In un articolo intitolato Parenting the Nation, Derek Wright, il maggior interprete di Farah, afferma che in Maps "the relations between a nation and its members are expressed through the roles of parents, or guardians, and children" (1992-1993: 177). 
su un'altra cartina mentale, la distanza incolmabile tra lui e Misra" (2003: 131). Ascar, il cui nome in somalo significa guerriero, esita tra la possibilità di combattere per la Somalia con le armi (cioè entrando nel fronte di liberazione) o con la penna (cioè sostenendo ideologicamente la battaglia dei somali) ${ }^{25}$. Il suo allontanamento da Misra diventerà definitivo soltanto quando la donna sarà accusata di aver tradito l'esercito somalo fornendo informazioni al nemico etiope. Pur in mancanza di prove certe, Misra verrà uccisa e il suo corpo sarà brutalmente mutilato.

Vediamo ora in che modo il romanzo di Farah tematizza le questioni introdotte grazie all'analisi del testo di Scego.

Quando il romanzo sta volgendo verso la fine, Askar e Hilaal, lo zio materno del ragazzo, hanno una lunga e cruciale discussione sulle mappe. Eccone un breve estratto:

E così iniziai a ridisegnare la mappa del corno d'Africa (Nella mia mappa l'Ogaden costituiva sempre parte integrante della Somalia). [...]

Lo zio fissò a lungo la mappa, trapassandola con il suo sguardo severo e concentrato. [...] Mi chiese: "Dimmi, Askar. Per te c’è verità nelle mappe che disegni? [...] È dalla tua anima che trai la verità inventata dalle mappe che disegni? [...] Il punto è questo: la verità cambia?” (2003: 285-6)

Se geografi, esploratori e conquistatori coloniali hanno smembrato o riunificato i popoli dell'Africa secondo criteri e mappe arbitrarie, allora il desiderio del ragazzo di reinventare quelle mappe affinché possano coincidere con i bisogni (culturali, psicologici, economici) suoi e del suo popolo sembra del tutto legittimo. Lo zio Hilaal, però, infonde il dubbio nel ragazzo, invitandolo a pensare che la verità non sia una sola né immutabile, che per i Somali "l'Ogaden come parte della Somalia è verità. Per un cartografo etiope, l'Ogaden come parte della Somalia è falsità" (288). Tuttavia il punto è un altro. Lo zio materno Hilaal - che, a differenza dello zio paterno Qorax, è un personaggio molto positivo: è colto, flessibile, comprensivo e persino capace, in una società profondamente patriarcale come quella somala ${ }^{26}$, di cedere a sua moglie alcune prerogative tradizionalmente maschili - se da un lato ricorda al nipote il valore posizionale della verità, dall'altro non è disposto, nemmeno lui, a mettere in discussione interamente il paradigma secondo il quale i somali sarebbero un popolo unito dalla lingua e diviso dalle mappe. Diversamente dagli abitanti dell'Etiopia (congerie di popoli, lingue, culture e tradizioni tribali), i somali - spiega Hilaal al ragazzo - sarebbero straordinariamente coesi da un punto di vista linguistico e culturale. Ecco perché la loro "verità" sull'Ogaden appare loro più legittima.

Ma l'identità somala è davvero individuabile ancora (se mai lo è stata) sulla base del criterio linguistico e culturale come vorrebbero il protagonista e i suoi zii? E allora perché Misra, che parla perfettamente somalo ed è imparentata a una famiglia somala è perennemente considerata straniera? Mentre altri somali, nominati nel testo, che vivono in Kenya, in altre zone dell'Africa, oppure nati in diaspora e che sono ormai incapaci di parlare correttamente somalo dovrebbero

25 Cfr. Colmer (2003).

26 La condizione della donna in Somalia è un tema che sta particolarmente a cuore all'autore. Cfr. in particolare From a Crooked Rib (1970), romanzo d'esordio, e Sardines (1981). 
continuare ad appartenere a questa comunità immaginata che è la Somalia? E ancora, perché il brutale Aw-Adan, pur non essendo di origine somale, può rivendicare la sua appartenenza alla comunità invocando l'identità religiosa (è infatti insegnante di arabo e di corano)?

Detto altrimenti, il romanzo ci induce a pensare che le mappe coloniali (e postcoloniali ${ }^{27}$, stabilite anch'esse sulla base di interessi spesso estranei alle aeree di riferimento), pur infondate e artificiose, abbiano avuto un effetto di verità non trascurabile. Le frontiere disegnate e ridisegnate dalle potenze europee (e in seguito, dopo il 1945, dalle superpotenze mondiali) hanno concretamente modificato la composizione etnica dei territori africani, per esempio dell'Ogaden, abitato ormai tanto da Somali quanto da Etiopi di varia origine etnica. Pertanto, qualsiasi tentativo di ristabilire una supposta "verità geografica" (come nel caso delle rivendicazioni somale in Ogaden) non può che sfociare in una violenza inaudita, come è dimostrato dalla storia di Misra, accusata - con buona probabilità ingiustamente - di aver tradito i somali durante la guerra. Più plausibile, invece, pensare che Misra abbia aiutato gli uni e gli altri, fornendo del cibo ai soldati etiopi e dei soldi ai somali. Ed è per questo che, nel sistema allegorico del romanzo, dove tutti i personaggi corrispondono a una porzione dell'identità o della società somala ${ }^{28}$, forse Misra, meglio di tutti gli altri, rappresenta la Somalia nel suo insieme: intrinsecamente plurale (non dimentichiamo la molteplice appartenenza etnica e culturale della donna), tiranneggiata da più padroni e infine brutalmente uccisa e smembrata. Benché il romanzo di Farah suggerisca, come quello di Scego, che le vicende individuali/familiari siano strettamente imbricate a quelle collettive/nazionali (perché l'una allegorizza l'altra e viceversa), Maps non elude, ma al contrario sottolinea la violenza sottesa a ogni rivendicazione di un "noi", perché ogni "noi”, avendo bisogno di pensarsi come coeso, per definizione cela e oscura le differenze che lo compongono.

Quanto ai problemi legati alla rappresentanza e alla narrazione di sé, Farah ci mette in guardia attraverso un uso accorto e raffinato di alcune strategie narrative squisitamente letterarie. L'aspetto più notevole del romanzo concerne la costruzione della voce del narratore, che oscilla continuamente tra la prima, la seconda e la terza persona, benché il testo rimanga sempre radicalmente focalizzato sulla figura di Askar. Il lettore è dunque costretto ad aderire al punto di vista del protagonista, per quanto Askar si riveli poco a poco un narratore inaffidabile. Questo spinge il lettore, soprattutto verso la conclusione, a dubitare di numerosi aspetti della storia raccontata dal protagonista: Misra ha davvero tradito l'esercito somalo? Chi ha assassinato la donna? Askar non ha forse partecipato all'omicidio rituale della sua madre adottiva? Chi racconta in seconda persona la storia di Askar? Questo narratore non corrisponde forse alla figura del giudice che interroga il protagonista?

Se è vero che pressoché tutti gli interpreti di questo romanzo ${ }^{29}$ hanno messo in rilievo il carattere inaffidabile del narratore, a mio avviso nessuno finora ha saputo trarre tutte le conseguenze di questa scelta narrativa. Il fatto che Askar sia un narratore inaffidabile credo abbia due implicazioni principali:

27 Il termine è qui usato in senso esclusivamente cronologico: il periodo che segue il crollo degli imperi coloniali.

28 Lo zio Halaal e sua moglie Salaado rappresentano la Somalia moderna europeizzata, il maestro Aw-Adan la Somalia musulmana coranica, Askar (un orfano affidato a una madre adottiva) l'Ogaden ecc.

29 Per una buona introduzione a Maps in particolare e alla produzione di Farah in generale, cfr. Ruggiero (1997), Wright (1994) e in particolare il poderoso volume curato da Wright (2002) che comprende quaranta saggi sull'opera di Farah. 
1) Se Misra è sia colei che custodisce il segreto del romanzo (soltanto lei potrebbe confermare il suo presunto tradimento e fare i nomi dei propri assassini) sia il punto di vista forcluso ${ }^{30}$ nel testo, dominato interamente dalla figura di Askar, allora il lettore è invitato continuamente a contro-focalizzare ${ }^{31}$, cioè a tentare di guardare attraverso gli occhi di Misra, che non può parlare perché è stata uccisa, e che non può scrivere perché è analfabeta.

2) Questo particolare esercizio di lettura tematizzato nel testo oltrepassa il romanzo in questione e finisce per estendersi alle letterature migranti e postcoloniali nel loro insieme. Askar, il quale esita tra la penna e le armi, è un probabile futuro scrittore che ipotizza un giorno di abbandonare la Somalia. Detto altrimenti, egli è un rappresentante dell'elite somala metropolitana esattamente come altri scrittori (somali) postcoloniali che usano l'inglese o altre lingue europee. Mettendo in dubbio la parola di Askar (che presuppone il silenzio di Misra), Nuruddin Farah mette indirettamente in dubbio anche la propria e quella degli altri scrittori postcoloniali, attraverso i quali i lettori occidentali conoscono i territori e le culture da cui i primi provengono. Più precisamente, il romanzo di Farah invita i lettori a riflettere sul fatto che lo scrittore migrante metropolitano, che ha accesso alla parola e alla pubblicazione in quanto presunto informante nativo, come è stato ben sottolineato da Spivak, è una delle figure di forclusione del/ della subalterno/a, cioè si comporta necessariamente come Askar nei confronti di Misra: egli parla al suo posto e la rappresenta, mettendo in scena la sua storia.

Il dubbio (sulle identità, sulle frontiere tra paesi, lingue e culture, sulla narrazione di sé, sul ruolo assunto dalle letterature postcoloniali nel mercato globale) è probabilmente il tema principale del romanzo, che non a caso inizia con la seguente epigrafe tratta da Socrate: "Si comincia a vivere quando si inizia a dubitare di tutto quello che ci ha preceduti".

\section{Riferimenti bibliografici}

Ashcroft, B., Griffiths, G., \& Tiffin, H. (1989). The Empire Writes Back: Theory and Practice in Post-colonial Literature. London-New York: Routledge.

Atkinson, D. (1995). Geopolitics, Cartography and Geographical Knowledge: Envisioning Africa from Fascist Italy. In: M. Bell, R. Butlin, \& M. Heffernan (Eds.), Geography and Imperialism (pp. 265-296). Manchester (NY): Manchester University Press.

Bertoni, C., \& Fusillo, M. (2001). Tematica romanzesca o topoi di lunga durata. In: F. Moretti (Ed.), Il romanzo. Temi, luoghi, eroi (vol. IV) (pp. 31-58). Torino: Einaudi.

Black, J. (1997). Maps and Politics. Chicago: University of Chicago Press.

Bonavita, R. (2009). Spettri dell'altro. Letteratura e razzismo nell'Italia contemporanea. Bologna: Il Mulino. Boddi, M. (2012). Letteratura dellimpero e romanzi coloniali (1922-1935). Minturno: Caramanica Editore.

30 Spivak prende in prestito dalla psicanalisi il termine "forclusione" e gli attribuisce un significato etico-politico: l'informante nativo è quell'alterità che l'episteme occidentale ha dovuto forcludere per potersi definire in quanto tale.

31 Sulla lettura come esperienza di controfocalizzazione (counter-focalization), cfr. Spivak (2002). 
Butler, J. (2006). Critica della violenza etica. Milano: Feltrinelli [ed. or. Giving an Account of Oneself. New York: Fordham University Press, 2005].

Casti, E. (2003). Les ateliers "culturels" de l'ailleurs: la cartographie de l'Afrique coloniale italienne. In: M. Colin, \& E. R. Laforgia (Eds.), L'afrique coloniale et postcolonile dans la culture, la littérature et la société italiennes (pp. 15-39). Caen: Presses Universitaires de Caen.

. (Ed.). (2007). Cartografia e progettazione territoriale. Dalle carte coloniali alle carte di piano. Torino: Utet Università.

Colmer, R. (2002). “This is a pen”: Writing in Maps. In: D. Wright (Ed.), Emerging Perspectives on Nuruddin Farah. (pp. 495-520). Trenton-Asmara: Africa World Press.

Conrad, J. (2007). Hearth of Darkness. Edited with an introduction by L. Piré. Firenze: Giunti.

Del Boca, A. (1984). Gli italiani in Africa orientale. Nostalgia delle colonie. Vol. IV. Roma-Bari: Laterza.

Dumoulié, C. (2012). Il soggetto postcoloniale. Dal pathos del "tra-due-io" all'esperienza del "tra-due-morti". Narrativa. Nuova serie, 33-34, 43-55.

Farah, N. (1970). From a Crooked Rib. London: Heinemann.

. (1981). Sardines. London: Allison \& Busby.

(2000). Rifugiati. Voci della diaspora somala. Roma: Meltemi [ed. or. 2000. Yesterday, Tomorrow. Voices from the Somali Diaspora. London, New York: Cassel].

_. (2003). Mappe. Milano: Frassinelli [ed. or. 1986. Maps. New York : Pantheon].

Giglioli, D. (2001). Tema. Firenze: La Nuova Italia.

- (2014). Critica della vittima. Roma: Nottetempo.

Guglielmi, M., \& Iacoli, G. (Eds.). (2012). Piani sul mondo. Le mappe nell'immaginazione letteraria. Macerata: Quodlibet.

Guglielmo, M. (2013). Il Corno d'Africa. Eritrea, Etiopia, Somalia. Bologna: Il Mulino.

Hardt, M., \& Negri, A. (2000). Empire. Cambridge-London: Harvard University Press.

Harley, B. (1988). Maps, Knowledge and Power. In: D. Cosgrove, \& S. Daniel (Eds.), The Iconography of Landscape (pp. 277-312). Cambridge: Cambridge University Press.

Huggan, G. (1989). Decolonizing the Map: Post-Colonialism, Post-Structuralism, and the Cartographic Connection. Ariel, 20 (4), 115-131.

- (1994). Territorial Disputes: Maps and Mapping Strategies in Contemporary Canadian and Australian Fiction. Toronto: University of Toronto Press.

Iacoli, G. (2007). Mappa, carta. In: R. Ceserani, M. Domenichelli, \& P. Fasano (Eds.), Dizionario dei temi letterari (pp. 1404-1407). Torino: Utet.

. (2008). La percezione narrativa dello spazio. Teorie e rappresentazioni contemporanee. Roma: Carocci.

Kuruvilla, G. (2008). È la vita, dolcezza. Milano: Baldini Castoldi Dalai.

. (2012). Milano, fin qui tutto bene. Roma-Bari: Laterza.

Mengozzi, C. (2013a). Narrazioni contese. Vent'anni di scritture italiane della migrazione. Roma: Carocci.

. (2013b). Archivio, mercato e strategie del vissuto. Su alcune scritture collaborative degli anni Duemila. In: M. Kleinhans, \& R. Schwaderer (Eds.), Transkurturelle italophone Literatur / Letteratura italofona transculturale (pp. 37-55). Würzburg, Königshausen \& Neumann.

Meneghelli, D. (2013). Storie proprio così. Il racconto nellera della narratività totale. Lodi: Morellini.

Pagliara, M. (1991). Il romanzo coloniale. In: G. De Donato, \& V. Gazzola Stachini (Eds.), I best seller del ventennio. Il regime e il libro di massa (pp. 365-80). Roma: Editori Riuniti. 


\section{- (2001). Il romanzo coloniale tra imperialismo e rimorso. Roma-Bari: Laterza.}

Pennac, D. (1993). La fata carabina. Milano: Feltrinelli [ed. or. 1987. La fée carabine. Paris: Gallimard].

Pinzuti, E. (2012). Sinthomatizzazione post-coloniale in Oltre Babilonia di Igiaba Scego. Narrativa. Nuova serie, 33-34, 43-55.

Proglio, G. (2011). Memorie oltre confine. La letteratura postcoloniale italiana in prospettiva storica. Verona: Ombre corte.

Quaquarelli, L. (2010). Rappresentazione del conflitto nella letteratura italiana della migrazione. Italies. Littérature. Civilisation. Société, 14, 401-410.

Ruggiero, R. (1997). Lo specchio infranto. L'opera di Nuruddin Farah. Pasian di Prato: Campanotto.

Rushdie, S. (1991). Imaginary Homelands. Essays and Criticism 1981-1991. London: Granta books-Penguin books.

Scego, I. (2005). Salsicce. In: F. Capitani, \& E. Coen (Eds.), Pecore nere (pp. 23-36). Roma-Bari: Laterza.

- (2008). Oltre Babilonia. Roma: Donzelli.

- (2010). La mia casa è dove sono. Milano: Rizzoli.

Scego, I., \& Bianchi, R. (2014). Roma negata. Percorsi postcoloniali nella città. Roma: Ediesse.

Sirotti, A. (2013). Riflessioni sulla lingua, retorica e stile in due autrici postcoloniali italiane: una letteratura maggiorenne? In: F. Sinopoli (Ed.), Postcoloniale italiano tra letteratura e storia (pp. 76-88). Aprilia: Novalogos.

Spivak, G. (1999). A Critique of Postcolonial Reason. Towards a History of the Vanishing Present. Cambridge: Harvard University Press.

- (2002). Ethics and Politics in Tagore, Coetzee, and Certain Scenes of Teaching. Diacritics, 3-4, $17-31$.

Tomasello, G. (2004). L'Africa tra mito e realtà. Storia della letteratura coloniale italiana. Palermo: Sellerio.

Wright, D. (1992-1993). Parenting the Nation: Some Observations on Nuruddin Farah's Maps. College Literature, 19-20 (3,1), 176-184. . (1994). The Novels of Nuruddin Farah. Bayreuth: Bayreuth African Studies. . (Ed.) (2002). Emerging Perspectives on Nuruddin Farah. Trenton-Asmara: Africa World Press.

Wood, D., \& Fels, J. (1993). The Power of Maps. London: Routledge.

Zammarano, V. T. (1934). Azanagò non pianse. Milano: Mondadori.

\section{Filmografia:}

Brioni, S., Guida, E., \& Chiscuzzu, G. (Dirs.) (2012). La quarta via. Mogadiscio, Pavia. Italia: Kimerafilm. 